\title{
Taranis: Neural networks and intelligent agents in the early warning against floods
}

\author{
Vivian F. López*, Santo L. Medina, Juan F. de Paz \\ Department of Computer Science and Automatics, University of Salamanca, Av. de la Merced 90, Salamanca, Spain
}

\section{A R T I C L E I N F O}

\section{Keywords:}

Neural networks

Intelligent agents

CouterPropagation

Early Warning System

SAT

Hydroelectric power

Dam

Agents

\begin{abstract}
A B S T R A C T
The following article proposes the implementation of a system based on neural networks of CounterPropagation type and intelligent agents for analysis and assessment of the risk of flood caused by rain, in addition to the implementation of agents in mobile devices for the presentation of alerts. Because as is known, natural phenomena have always existed, but in recent years as a result of global warming we have seen that floods are becoming more frequent, which has forced the creation of specialized agencies and intelligent mechanisms to prevent the loss of human lives due to these phenomena.
\end{abstract}

(C) 2012 Elsevier Ltd. All rights reserved.

\section{Introduction}

The Early Warning System (EWS) is the first line of defence for the most vulnerable population against large-scale natural catastrophes. This is the main reason for the development of this application Taranis, which uses artificial intelligence in a hydroelectric structure to provide a better source of information, when the data of the threats are already known. With this type of system, if corrective measures were taken in time, these catastrophes could be avoided. If theses preventive measures are not adopted flooding will occur, as was the case in the Dominican Republic caused by the tropical storm Olga in 2007, which was based on an outdated operations manual and limited to a specific period of time for storms, without taking climate change into account and not taking the decision to empty the reservoir in time, which caused the floods in Santiago de los Caballeros in December 2007.

The greatest deficiency in the current EWS is the mechanisms to alert the public in a rapid, accurate and efficient manner. It is no use having the best prediction system if there are not any mechanisms to alert the population in case of emergency. At the present time warning mechanisms on a large scale are carried out through short text messages to mobile phones. The most representative of this practice is the United States and Japan. In addition, Chile has recently begun the implementation of this technology.

In this article we present a tool that has as its objective to use the capacity of the neural network (NN) pattern recognition, to predict the volume of precipitation expected at any given time, information that would be used to create models to predict floods and provide information to manage the hydroelectric reservoir in an effective manner to prevent material damage. Another advantage of NNs used by this system is the ability to adapt to

\footnotetext{
* Corresponding author. Tel.: +34 667702837.

E-mail address: vivian@usal.es (V.F. López).
}

the new meteorological values caused by climate change. On the other hand, the system provides an alternative means of communication and issuance of massive alerts, through the use of intelligent agents, which is prepared to handle earthquake, tsunami and wildfire alerts, although the current system focuses on alerting against flooding.

\subsection{Early warning systems}

The EWS is a tool that consists of a set of mechanisms and procedures for the detection of hazards, monitoring of indicators, communication of alerts and alarms and evacuation of vulnerable populations to safe areas (Lyon \& Fletcher, 2001).

The EWS is made up of institutions that are responsible for assessing natural phenomena through the instrumentation and broadcasting of alerts to ensure that the institutions of civil society are responsible for communicating to the civilian population of the possibility of such an extreme phenomenon occurring and proceed with the evacuation or procedures to mitigate the losses.

The EWS requires technical knowledge about the threat, what the causes are and the risks faced by society. This involves knowing the temporal and spatial behaviour of such phenomena through the modelling of physical quantities and the development of procedures to make the forecasts that are applied as part of the EWS. In this context, one looks for precursor signals that may relate to the likely magnitude of the event.

The issue of the EWS is one of the most commented on today. International agencies and other developed countries have been trying to prevent disasters and not only limit themselves to help once they have happened. We have knowledge of floods since the beginning of civilization, but as a result of climate change these are becoming increasingly frequent and aggressive, a situation which has led to the main organizations for international cooperation to search for alternatives to preserve life and property. This is 
the reason why the area of Central America and the Caribbean currently have 19 EWS against floods of a centralized type and 26 of a decentralized or community type, and the number continues to rise.

EWS requires 4 basic components (ISDR, 2006), these are:

1. Knowledge of the risks,

2. Technical monitoring and alert service,

3. Communication and dissemination of the alerts,

4. The ability of community responses.

The community EWS as well as the centralized one possess a great deficiency in the general structure. While the forecasts have improved the way information is structured to meet requirements, it turns out to be very expensive and the most of the cases do not have the 4 elements that an EWS should have.

These systems are implemented with fixed data structures and do not have the capacity to use new technological advances; on the other hand, the platforms are closed which increases the costs of implementation and maintenance; another factor that elevates the cost is that of communication platforms which are based on radio.

Another deficiency that the EWS has is that these systems are mostly used against the threat of flooding, while these countries have at least 3 other different risks, such as: tsunamis, earthquakes and volcanic eruptions. Likewise, they do not have mechanisms to alert the entire population of an area or region at the same time; the alerts and warnings are directly implemented by relief agencies.

On the other hand, the ability to integrate of different data sources is limited. In most cases they are based on forecasts of foreign agencies, mainly due to the scepticism meteorological organizations have of the countries concerned in the Central American area and the Caribbean.

The knowledge of the risk and technical monitoring are the two basic components of an EWS, for which Taranis use NN to predict floods, and management of hydroelectric dams. It also offers intelligent mechanisms for communication and dissemination of mass alerts through agents, which are also supposed to control the reading of the hydrometeorological stations, with which the third structural of this type of system requirement is met and, likewise, through the knowledge of the level of risk of each community to evaluate the main requirements for each case. With the communication mechanism previously mentioned, you will obtain better control of the evacuation operations.

\section{Neural networks}

NNs applied to solve a variety of problems, according to Freeman and Skapura (1992), is a system of parallel processors connected to each other in the form of directed graph. Schematically each neuron of the network is represented as a node. These connections provide a hierarchical structure that attempts to emulate the physiology of the brain, searching for new models of processing to solve specific problems in the real world. What is important in the development of the technique of the NN is the useful behaviour to be learned, recognized and applied between objects and frames of objects in the real world. In this way, the NNs are used as a tool to resolve difficult problems.

Several types of NNs are used to solve prediction problems. These NNs models use supervised learning algorithms. As examples the BackPropagation model (BP), Radial Basic Function (RBF) and the CounterPropagation network (CPN) can be cited (RongKwei \& Tzu-Chiang, 2005). These models are used because in most cases recognizing patterns is required to identify when these facts or phenomena will occur again before they happen.
The CPN was introduced by Hecht Nielsen (1987) and presents two types, full and forward-only. The CPN was developed to provide an efficient method of approximation for a function $y=f(x)$. The full CPN works best only when there is an inverse function $f^{-1}$. Forward-only, designed to achieve an approximation to $y=f(x)$ when $f^{-1}$ is not required. The architecture of the forwardonly consists of three layers: input, hidden (Grossberg, 1987; Kohonen, 1990). The input layer with $n$ nodes stores the input values, the Kohonen layer (hidden) with $p$ cluster calculates the similarity between the input values and the Grossberg layer with $m$ nodes calculates the output and computes the weighted sum of the output of the Kohonen layer (Chang \& Chen, 2001).

The CPNs have a learning hybrid between the self-organizing map (SOM) (Kohonen, 1990) and the feed forward neural network (FFNN) supervised learning network (Lippmann, 1987), and this hidden layer is trained using the INSTAR algorithm and the FFNN is trained using OUSTAR algorithm. The Fig. 1 shows the architecture of the CPN network.

The operation of the learning of the layers, is as follows: the input layer data must be normalized or scaled to fit the calculations, and this is achieved with the following equation:

$I=\frac{x}{\|x\|}=\frac{x}{\sqrt{\sum_{j=1} x_{j}^{2}}}$

where $I$ is the weight for each input $x$. Then, each element of the hidden layer is calculated as:

Neta $=I * W$
Neta $=\|I\| *\|W\| \cos (\Theta)$
Neta $=\cos (\Theta)$

In this selection process a vector of the input layer is chosen at random, then normalized and entered into the network. The winning vector is updated according to Rumelhart and Zipser (1985).

$\Delta W_{i j}= \begin{cases}\alpha\left(X_{i}-W_{i j}\right) & \text { winning node } \\ 0 & \text { otherwise }\end{cases}$

where $\alpha$ is the learning rate, if $\alpha>0, X_{i}$ is the ith node of input vector, and $W_{i j}$ is the weight of the ith input node to the winner node $j$. The competitive signal, which is a binary variable assuming a value of one for the winning node and a value of 0 in all other cases, sent from the winning node to the Grossberg layer is 1 , and the competitive signals sent by the other Kohonen nodes are 0 .

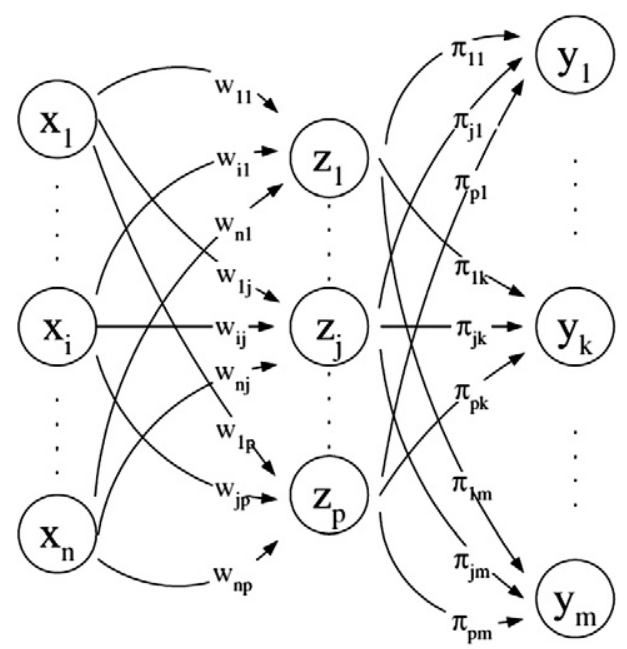

Input layer Kohonen layer Grossberg layer

Fig. 1. CPN architecture. 
After the weight vector from the input layer to the Kohonen layer has been determined, the weight vector between the Kohonen layer and the Grossberg layer are trained by:

$\Delta \pi_{i j}=Z_{j} \beta\left(Y_{k}-\pi_{i j}\right)$

where $Z_{j}$ is the competitive signal, $\beta$ is the learning rate, $Y_{k}$ is the target and $\pi_{i j}$ is the weight of the Kohonen node $j$ to Grossberg node $k$. The output of the node $k$ is given by:

$y_{k}^{*}=\sum_{j=1}^{p} \pi_{j k} Z_{j}$

where $y_{k}^{*}$ is the calculated output.

After the CPN is trained, all of the weight will be fixed. Only input data is needed for the model to operate when the CPN is used for predicting.

\section{Taranis application}

The development of the application uses the concept of systems-based agents, because this requires the same to be implemented without the obligatory nature of agents, and in this way take advantage of the fundamentals of both paradigms (systemsbased agents and multi-agent systems) (Corchado, Tapia, \& Bajo, 2012).

\subsection{Application of the CPN networks in the prediction of rain}

\subsubsection{Telemetric network}

For the implementation of the system it has a telemetry network consisting of 5 stations located on the river of the Yaque del Norte (Indhri station, 2010). Table 1 lists the stations, as well as the geographical position which are as follows.

For the training and testing of NN a data set of was used. The data for the verification had 9300 records with the values at measurement hydrometeorological stations at the basin of the River Yaque del Norte in the Dominican Republic. Each record contains the measurement values of temperature, wind, wind direction, relative humidity, solar radiation and atmospheric pressure.

The NN scheme for forecasting the volume of expected rain is as shown in Fig. 2. This has an entry layer with 6 neurons, corresponding to the hydrometeorological variables; a hidden layer with 30 neurons and an output layer with values that corresponds to the volume of corresponding rain.

In the original scheme of the CPN the activation function of the hidden layer uses the competitive function, but this project was modified to a sigmoid function because the resilient propagation algorithm cannot be used with the competitive activation function. This function can only be used when positive output values are expected because it only produces positive outputs (Heaton, 2010). The equation for this function is:

$f(x)=\frac{1}{\left(1+e^{x}\right)}$

The graph scheme of the activation function is shown below in Fig. 3.

Table 1

List station of network telemetry.

\begin{tabular}{llll}
\hline No. & Station & Latitude & Longitude \\
\hline 1 & Jarabacoa & 331100 & 2115150 \\
2 & Manabao & 311130 & 2108700 \\
3 & Santiago & 316800 & 2150750 \\
4 & Tavera & 320450 & 2132350 \\
5 & Mata Grande & 290800 & 2124650 \\
\hline
\end{tabular}

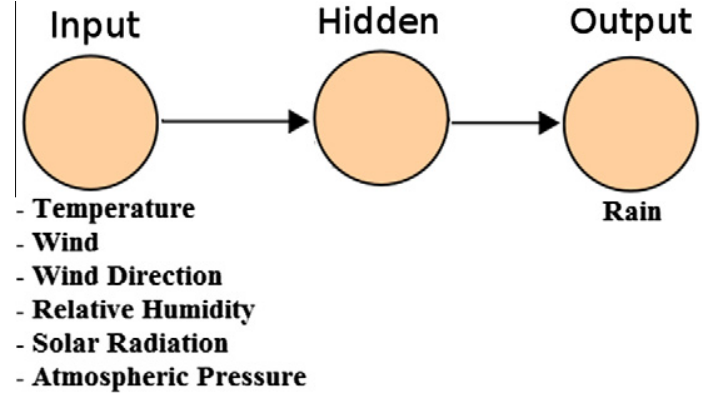

Fig. 2. Neural network for forecasting.

\subsubsection{Reservoir network}

A second layer of the NN is responsible for determining the evolution of the reservoir:

In Fig. 4 is the scheme of the NN to determine the incidence of rainfall in the reservoir. This has an entry layer with 3 neurons, corresponding to the variables of the predicted volume of rain, volume of entry, and level of the reservoir; a hidden layer with 9 neurons and a layer with an output value that corresponds to the volume that can be emptied.

\subsubsection{Function activation competitive}

For the neurons in the reservoir layer activation competitive was used for the original scheme of neurons. This function is only used to force the selection of a group of winning neurons. The winning group of neurons is the one with the highest output. This function first determines the winning neurons. Losing neurons are matched to zero. The winners may be of equal value. At the beginning of the function all neurons selected as winners are tracked. This uses a function of derivation which receives a double as a parameter (Heaton, 2010). The graphic scheme of the competitive activation is shown in Fig. 5:

The activation function of the input and Grossberg layer use the linear activation function (Heaton, 2010). This uses a linear equation, which is shown in Fig. 6:

$f(x)=x$

Below is the graph of the linear function (Heaton, 2010).

\subsection{Structure of the application}

The application's architecture is based on layers, each independent of the other which makes it highly scalable and interoperable.

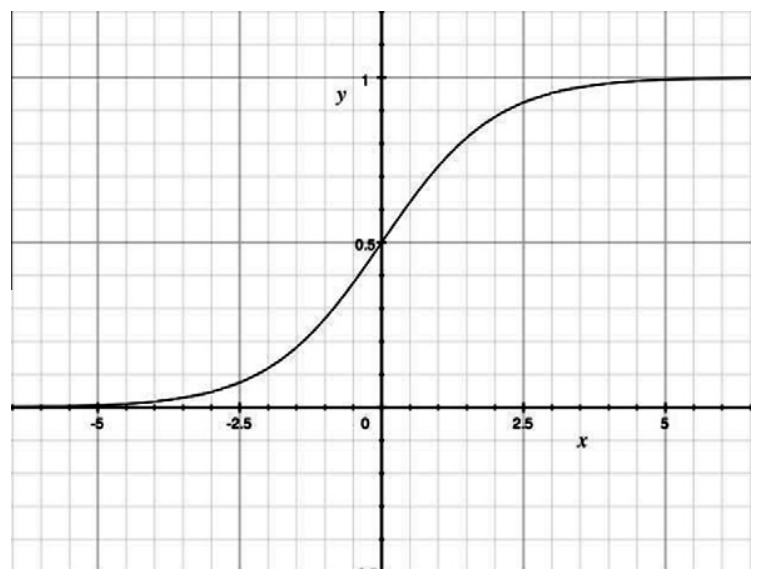

Fig. 3. Sigmoid activation function scheme. 


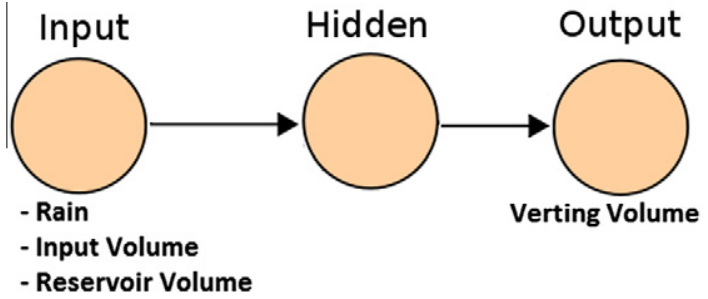

Fig. 4. Neural network for reservoir.

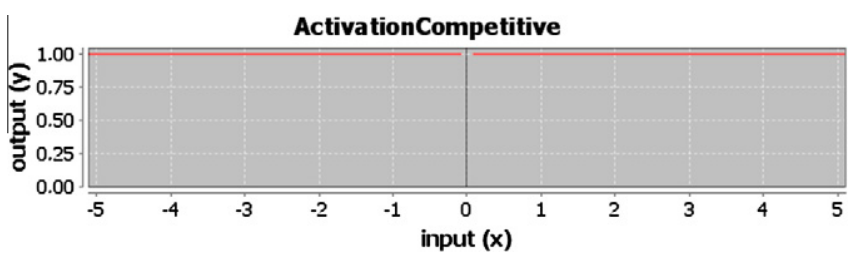

Fig. 5. Competitive activation function scheme.

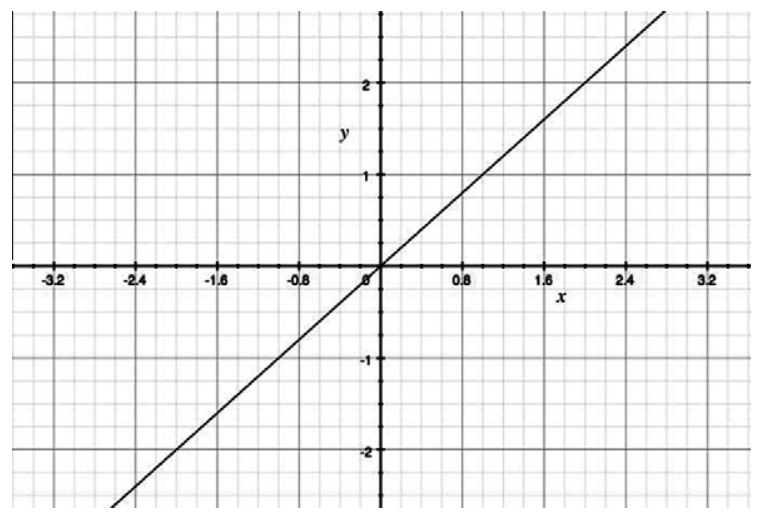

Fig. 6. Linear activation function scheme.

Each layer is responsible for performing a function which together complement the system. The structure is based on a modification of the model of three layers of software architecture; these are: data layer, services layer (Web, sensors) and presentation layer (Web, Windows and agents).

Two graph interfaces based on Windows and Web are necessary because due to the time that the training of the network requires, it is not possible to do it in a Web environment.

In Fig. 7, shows the diagram of the application, and in this the sensors of the weather stations are read by the agents and the information is sent to the server database through the web service. This information is processed by the server model and if there is a level of warning, it notifies about the possibility of danger. If you reach the level of alert allows you to publish the alert in the web application and send alerts to mobile devices that have agents and which are specified.

In addition to data from the sensors model generation application, it loads the data from the NOAA (NOAA, 2009) files containing the prediction about the rainfall expected in $6 \mathrm{~h}$ and relates these two data source with data from the SCADA which provides information on the levels of the central and the volume of incoming water to the reservoir.

Likewise, from the implementation of model generation you can acquire information from the states of the sensors of the stations, and check the state of the agents.

\subsection{Intelligent agents in mobile application}

The technology used by some countries issuing alerts is cell broadcast services (CBS). This technology presents several problems, the main one being the cost of implementation. Therefore, on the one hand, it is necessary to expand the capacity available to the telephone companies for the sending of mass messages. Another drawback is that it requires special operation companies.

The mobile alert system is based on intelligent agents that are incorporated into equipment with Android Operating Systems, but can be incorporated into equipment with Connected Device Configuration (CDC) or Mobile Information Device Profile (MIDP) Java and Windows Mobile. The implementation of this platform would eliminate another drawback of the Short Message ServiceCell Broadcast (SMS-CB) technology, which is the limitation of 160 or 170 characters in messages. Presented in this way it is a more usable platform and with features for people with disabilities who can take advantage of technological advances in mobile telephony, such as the incorporation of new sensors.

Although the application of the NN presented in this paper corresponds to a flood warning EWS, the mobile application is ready to handle other types of alerts such as earthquakes, tsunamis or forest fires. Fig. 8, shows the types of alert managed.

It is necessary to indicate that mobile agents can be upgraded to expand the options available for users and can use new technologies that can be incorporated to mobile phones, for example, the reading of Bluetooth or WI-FI sensors. It even offers the possibility of interaction with other intelligent agents and work in a collaborative way.

This version of the application is available in the following languages; English, Spanish, French, Italian, Japanese, Portuguese.

\subsection{Alert levels}

It is the level that represents the degree of certainty of an extreme event occurring. In the implementation of alerts, in addition to images, they use colors to further help the user to discern the level of alert that is being shown. For this purpose the following scheme has been established.

1. Normal (Green or Grey)Status of a normal situation.

2. Warning (Orange)

The basin has a sign of abnormality in the channel or existence of the possibility of an event that could cause an emergency and require a warning to the User Control type to start the process of emergency. In some phenomena such as earthquakes, it can pass from Grey to Red Alert, due to the characteristics and the greater need for speed in response.

\section{Alert (Red or Brown)}

Confirmation of the emergency, beginning of the recovery process before disasters.

\subsection{Usability options}

The application presents an icon at the top, indicating the status of the application at all times. In addition, to achieve a correct display, the images have been defined for three screen sizes, depending on the dpi of the same which are High Dots Per Inch (HDPI), Medium Dots Per Inch (MDPI), Low Dots Per Inch (LPDI) (Android developers, 2011).

The colors selected have been chosen taking into consideration the colors that can be seen by persons with disorders in the identification of the colors (colorblind people). According to the research on the problems of colorblind people there are two major groups, those who confuse red and green colors, and those who cannot distinguish blue. 


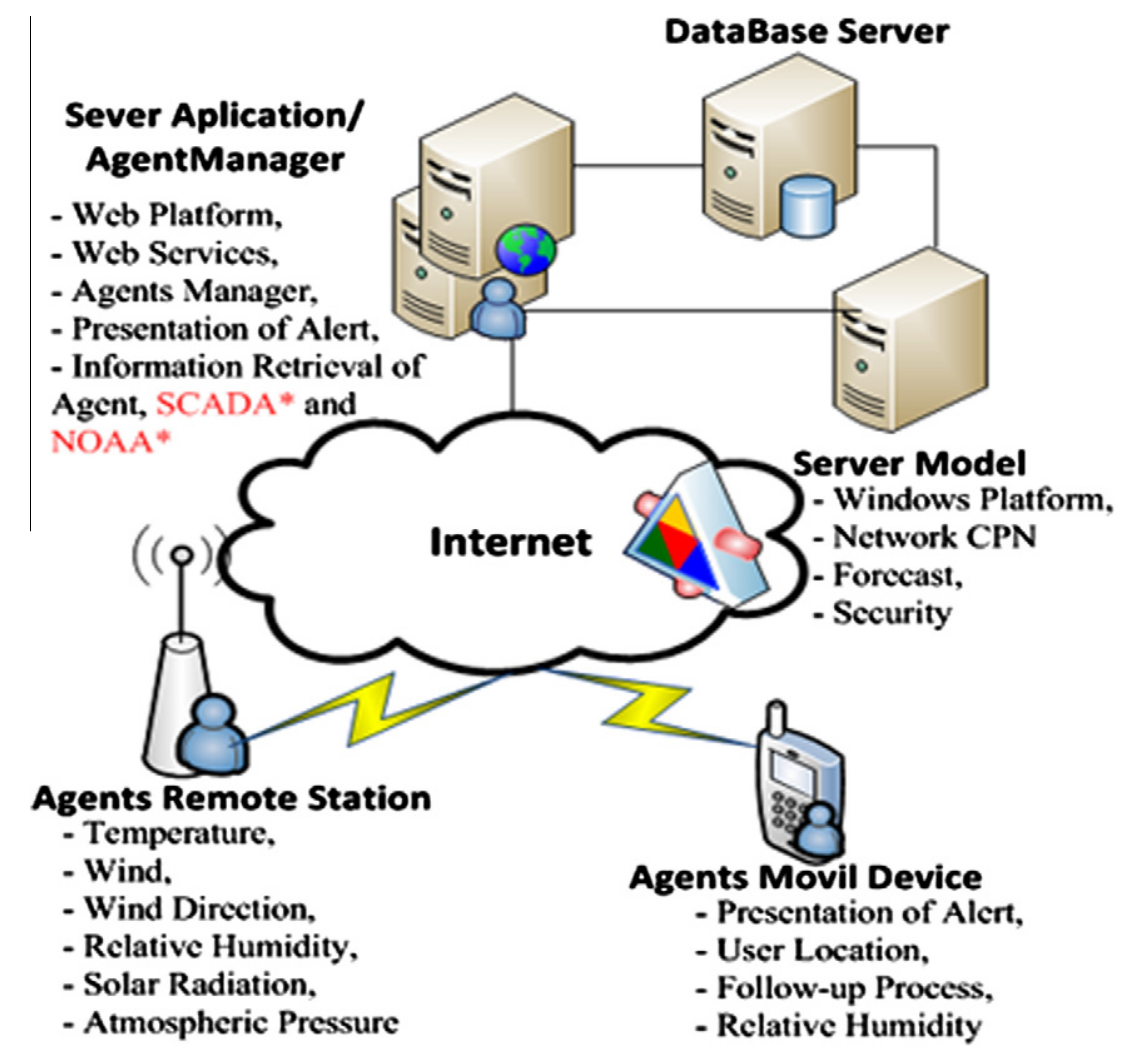

Fig. 7. System architectures.


Fig. 8. Types of alerts available.

Being aware that persons with disabilities should be taken into account in moments of warnings and alerts. In addition to modifying colors of alert level standards so that people with color recognition problems can efficiently receive messages, functions to translate the text content to voice messages and an outline of vibration will be incorporated. Thus people with visual disabilities can be kept informed.

\section{Experiments and result}

The first evaluations of the NNs were carried out using the data from the hydroclimatic stations in Santiago, Taveras, Jarabacoa and Manabao, which are located in the basin of the Yaque del Norte river. Data was extracted from the information system in real time the Instituto Nacional de Recursos Hidraulicos (INDRHI) in the Dominican Republic (Indhri station, 2010), but due to the fact that the daily sequence was relatively short and that after the pre-processing the information available was reduced considerably, as well as becoming inconsistent for a proper climate learning, much of the daily sequence could not be used in some cases due to the absence of full or partial values.

A second set of data used for the tests was that from the Chama station (2011) located to the south of Lake Maracaibo, in Venezuela a station with a climate and longitude similar to that of the original stations. These data corresponded to the measurements taken every $30 \mathrm{~min}$, from August 2001 to July 2005 (Chama station, 2011). This data set did with the record of $173 \mathrm{~h}$.
A third set of data used came from the meteorology station of Audubon located in Tucson, Arizona (Agricultural research services, 2011). This data corresponds to the measurements taken every 20 min between 19 January 1997 to December 5, 2006. The Table 2 shows in detail the distribution of the data.

The other data from evaluated stations correspond to the Mucujún station (2011), La Hechicera station (2011) stations, but were discarded for being at a much greater altitude than the stations whose data will be used by Taranis.

The CPN was trained using the Ecocog tool (Heaton, 2010), a NN framework for Java and .NET. For the training, we consider an maximum error of $1 \%, 50$ steps as maximum and an initial update value of 0.1 . Data that was managed to reach to the set of training threshold expected in only 84 iterations in $25 \mathrm{~s}$. The initial error percentage was $7 \%$, with a variation of average error of 0.06947342 . The Fig. 9 shows the evolution of the learning process of network.

After the tests with data from different areas and climates, it was determined that for tropical areas, depending on the characteristics of the basin, $20 \mathrm{~min}$ is the ideal time for the reading of the measurements (instead of $1 \mathrm{~h}$ which is the average in most of the stations), during the occurrence of unusual rain or for rains which exceeds the normal parameters. This process is extremely easy to perform with Taranis, which can be configured to automatically fit the change the sensor reading times, then that is detected the sudden increase in the rainy season.

Table 2

Description data test.

\begin{tabular}{lll}
\hline Data type & Station audubon & Error \\
\hline Training data & 86,468 & 0.009979 \\
Evaluation data & 37,059 & 1.005134 \\
\hline
\end{tabular}




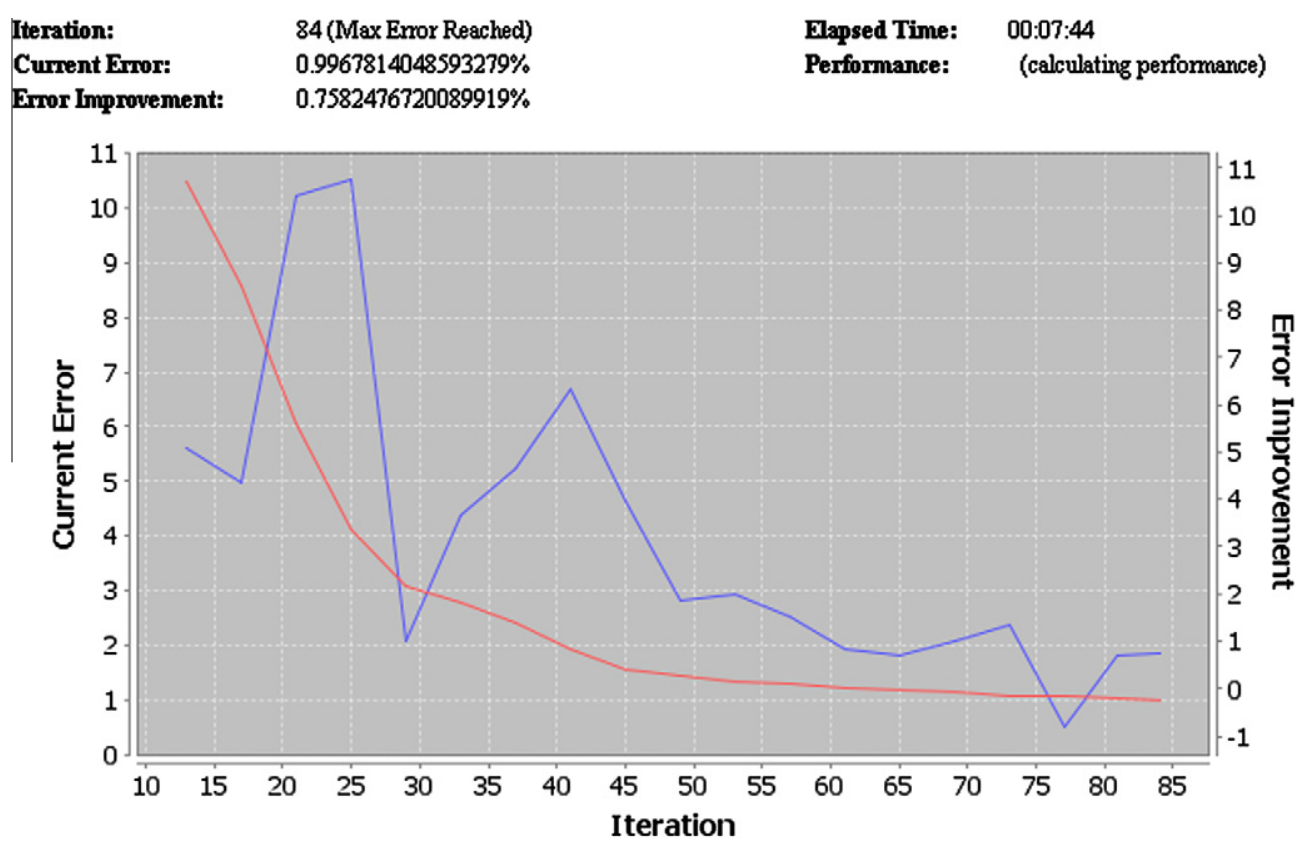

- Current Error - Error Improvement

Fig. 9. Process training network

\section{Conclusions}

In this article we presented a EWS that use NNs of CounterPropagation type and intelligent agents for analysis and assessment of the risk of flood caused by rain. The system creates models to predict floods and provide information to manage the hydroelectric reservoir in an effective manner to prevent material damage. The use of NNs provides an interesting alternative to adapt to the new meteorological values caused by climate change. On the other hand the addition of a second layer of NNs, to assess the level of the reservoir, would allow the operation of the central authorities to take the appropriate decisions at the right time. With the results described in Section 4 it is clearly shown that with the Nns classifiers a high level of accuracy can be achieved.

Taranis also offers intelligent mechanisms for communication and dissemination of mass alerts through agents, which are also used to control the reading of the hydrometeorological stations, with which the third structural of this type of system requirement is met and, likewise, through the knowledge of the level of risk of each community to evaluate the main requirements for each case. With the communication mechanism previously mentioned, you will obtain better control of the evacuation operations.

On the other hand, the system also provides an alternative means of communication and issuance of massive alerts, through the use of intelligent agents, which is prepared to handle earthquake, tsunami and wildfire alerts, although the current system focuses on alerting against flooding.

The persons with disabilities are taken into account in moments of warnings and alerts. In addition to modifying colors of alert level standards so that people with color recognition problems can efficiently receive messages, functions to translate the text content to voice messages and an outline of vibration will be incorporated. Thus people with visual disabilities can be kept informed with Taranis.

\section{Future applications}

For a second implementation of the network layer scheme two new networks levels will be added, which will attempt to predict the evolution volume at the front and rear of the reservoirs. What is achieved is having more control and optimizing the process of drainage of hydroelectric dams, in addition to being able to improve the way in which resources are used in the event of drought. Likewise, enabling access to satellite information and the establishment of a hydrological database are intended.

Also, intelligent agents are created with embedded neural networks to act with the warning and alert process in an autonomous way, in the event that communication with the central application fails. On the other hand, it increases the communication options with people who have other types of disabilities, which have not been included in the first version.

\section{References}

Agricultural Research Services, (2011). USDA ARS, <http://www.tucson.ars.ag.gov/ metdap/metdata.aspx>.

Android developers. (2011). Supporting multiple screens, <http://developer android.com/guide/practices/screens-support.html $>$.

Chama station, (2011). <http://www.cecalc.ula.ve/redbc/estaciones/estacion chama.html>.

Chang, F. J., \& Chen, Y. C. (2001). A counterpropagation fuzzy-neural network modelin approach to real time streamflow prediction. Journal of hydrology. http://dx.doi.org/10.1016/S0022-1694(01)00350-X.

Corchado, J. M., Tapia, D. I., \& Bajo, J. (2012). A multi-agent architecture for distributed services and applications. International Journal of Innovative Computing, Information and Control (IJICIC), 8(4), 2453-2476. ISSN: 0824-7935.

Freeman, J. A., \& Skapura, D. M. (1992). Neural networks algorithms, applications, and programming techniques. Addison Wesley.

Grossberg, S. (1987). Competitive learning: From interactive activation to adaptive resonance. Cognitive Science, 11, 23-63.

Heaton, J. (2010). Introduction to Encog 2.5 for Java, Heaton Research.

Hecht Nielsen, R. (1987). Counterpropagation networks. Applied Optics, 26(23), 4979-4983. http://dx.doi.org/10.1364/AO.26.004979.

Indhri station, (2010). <http://www.indhri.gob.do>.

ISDR. (2006). EWC III Tercera Conferencia Internacional sobre Alerta Temprana, Desarrollo de Sistemas de Alerta temprana, In International strategy for disaster reduction (ISDR).

Kohonen, T. (1990). The self-organizing map. IEEE.

La Hechicera station. (2011). <http://www.cecalc.ula.ve/redbc/estaciones/estacionla-hechicera.html>.

Lippmann, R. P. (1987). An introduction to computing with neural nets. IEEE ASSP Magazine, 4, 4-22.

Lyon, G. R., \& Fletcher, J. M. (2001). Early warning system. Education Matters, 1(2), $2-29$. 
Mucujún Station, (2011). <http://www.cecalc.ula.ve/redbc/estaciones/estacion_ mucujun.html>.

NOAA (2009). Automated eTRaP Information, <http://www.ssd.noaa.gov/PS/TROP/ etrap-info.html>
Rong-Kwei, L., \& Tzu-Chiang, L. (2005). A new ART-counterpropagation neural network for solving a forecasting problem. Expert Systems with Applications. Rumelhart, D., \& Zipser, D. (1985). Feature discovery by competitive learning. Cognitive Science, $7,75-112$. 